Annales Geophysicae (2002) 20: 511-521 (c) European Geophysical Society 2002

\title{
The method of determination of the long-term trends in the F2 region independent of geomagnetic activity
}

\author{
A. D. Danilov \\ Institute of Applied Geophysics, Rostokinskays 9, Moscow 129 128, Russia \\ Received: 10 May 2001 - Revised: 1 October 2001 - Accepted: 21 November 2001
}

\begin{abstract}
A detailed analysis of the $f o \mathrm{~F} 2$ data at the Sverdlovsk station is performed to answer the question: whether the long-term trends of the F2-layer parameter detected recently are mainly due to the long-term changes in geomagnetic activity during the recent decades. Two methods to derive trends independent of geomagnetic activity are developed. It is found that both methods agree well and give a relative nongeomagnetic trend of about -0.0015 per year (or an absolute nongeomagnetic trend of about $-0.015 \mathrm{MHz}$ per year). The close relation of $f o \mathrm{~F} 2$ to $A_{p}$ found by several authors is mainly due to a high correlation between the $f o \mathrm{~F} 2$ deviations from the regression line and year-to-year changes in the $A_{p}$ index rather than to systematic long-term changes of $A_{p}$ as has been suggested earlier.
\end{abstract}

Key words. Ionosphere (ionospheric distances; modeling and forecasting)

\section{Introduction}

Studies of the long-term changes (trends) in the parameters of the upper atmosphere and ionosphere are currently very popular. Several groups of authors (Bencze et al., 1998; Bremer, 1996, 1998, 2001; Danilov and Mikhailov, 1998, 1999, 2001; Givishvily and Leshchenko, 1993, 1994; Jarvis et al., 1998; Marin et al., 2001; Mikhailov and Marin 2000, 2001; Ulich and Turunen, 1997; Ulich et al., 1997; Upadhyay and Mahajan, 1998) studied trends of the F2-layer parameters, $h m \mathrm{~F} 2$ and $f o \mathrm{~F} 2$. The results of these studies differ significantly by the methods of trend identification used and the results obtained. To analyze and review all of these studies is not the goal of this paper; rather, this paper is aimed at a discussion of the relatively recent problem of the relation between the F2 parameter trends and geomagnetic activity.

Danilov and Mikhailov $(1998,1999)$ were the first to attract attention to the fact that the trends of the critical frequency $f o \mathrm{~F} 2$ obtained at different stations demonstrate a de-

Correspondence to: A. D. Danilov (adanilov99@mail.ru) pendence on the station geomagnetic latitude $\Phi$, decreasing with a decrease in $\Phi$. This was an important starting point of the concept that the trends observed are related to the changes in geomagnetic activity during the recent decades. Mikhailov and Marin (2000) demonstrated that the annual mean deviations $\delta f o \mathrm{~F} 2$ from the regression line in terms of the solar activity index R12 correlate well with the geomagnetic index $A_{p}$. The observed values of $\delta f o \mathrm{~F} 2$ repeat the time behaviour of $A_{p}$ : they decrease until approximately 1965 and then increase in the 1965-1991 period.

Danilov and Mikhailov (1998, 1999, 2001) and Mikhailov and Marin $(2000,2001)$ noted that the relative $f o \mathrm{~F} 2$ trends, $k$, obtained demonstrate seasonal and (especially) diurnal variations, which indicate a realization mechanism of the relation found between the $A_{p}$ and $\delta f o \mathrm{~F} 2$ trends. It was suggested (Danilov, 2000; Danilov and Mikhailov 2001, Mikhailov and Marin, 2000, 2001) that the $f o \mathrm{~F} 2$ trends observed during the recent decades are caused by the positive trends (an increase) in the number (and/or intensity) of ionospheric disturbances (negative phases of the so-called ionospheric storms accompanying, as a rule, geomagnetic storms). Based on the experimental data analysis, corresponding trends of these ionospheric storms were derived (Sergeenko and Kuleshova, 1994; Sergeenko and Givishvili, 1997).

Danilov and Mikhailov (2001) studied in detail the problem of the relations of the $f o \mathrm{~F} 2$ trends to geomagnetic activity based on the data of two Southern Hemisphere stations, Argentine Islands and Port Stanley. The most important conclusion was that the correlation coefficients between $\delta f o \mathrm{~F} 2$ and $A_{p}$ are maximum and significant in the very hours of the day when the occurrence of the negative phase of an ionospheric storm is most probable. Thus, the concept was born that all (or, at least, the majority) of the F2-layer parameter trends detected in the recent publications are a manifestation of the trend (the increase) of geomagnetic activity from the mid-sixties to the beginning of the 1990s (this period covers most of the time intervals for which the $f o \mathrm{~F} 2$ data for many stations were analyzed). 
Taking into account the above cited papers, the impact of the geomagnetic activity changes (to describe these we use here the anual mean value of $A_{p}$ ) on the observed trends of $f o \mathrm{~F} 2$ seems undoubted. However, the relative significance of geomagnetic activity and ionospheric storms against other drivers in inducing observed trends of $f o \mathrm{~F} 2$ and $h \mathrm{mF} 2$ is not yet clear. The question is not answered, as to whether there are long-term changes (trends) in the F2 layer not related to $A_{p}$ variations. To avoid taking extra responsibility, we will call them simple "nongeomagnetic" trends, though probably these trends, if they do exist, have the anthropogenic origin.

There are several reasons for the possible existence of nongeomagnetic trends. First of all, there are widely known publications from the beginning of the 1990s (Rishbeth and Roble, 1992; Rishbeth, 1990) which evaluated expected changes in the upper atmosphere and ionosphere due to the increase in the amount of greenhouse gases. Second, during the 1990s, a lot of publications appeared (see e.g. the review by Danilov, 1997) indicating strong trends in the upper mesosphere and lower thermosphere parameters (first of all, the temperature). If even the moderate estimates of the effect are correct, one may hardly expect that there would be no response to these trends at F2-region heights. And third, last but not least, during the recent decade, publications appeared (see e.g. the monograph by Adushkin et al., 2000) describing a huge amount of admixtures released into the upper atmosphere in the course of launching and operating various space vehicles. Evidently, due to the small neutral gas density, the upper atmosphere is very vulnerable and there may be observed anthropogenic contaminations (and so anthropogenic trends) different, and probably stronger, than those in the lower atmosphere.

To develop a method to reveal nongeomagnetic trends in $f o \mathrm{~F} 2$ on the background of the variations of this parameter with geomagnetic activity, the data on $f o \mathrm{~F} 2$ measured at the Sverdlovsk station were analyzed in detail. The choice of this station was due to several reasons: the station is located at middle geographic and geomagnetic latitudes $\left(\varphi=56.7^{\circ} \mathrm{N}\right.$ and $\Phi=48.4^{\circ} \mathrm{N}$ ), is known for its reliable measurements, fits well the picture of the geomagnetic activity impact created in the papers described above, and finally, the period of observations available (1948-1994) is convenient for the analysis, because it includes periods of both decreasing and increasing geomagnetic activity (see below). Variations in the annual mean value of $A_{p}$ for the entire period considered are shown in Table 1. One can see that the first 15-17 years of the period are characterized by a systematic decrease in $A_{p}$ with time, whereas the last 20-25 years fall on a systematic increase in $A_{p}$, which has already been mentioned above.

In order to look for $f o \mathrm{~F} 2$ nongeomagnetic trends, the entire period from 1948 to 1994 was split to 18 running intervals, each 30 years long: 1948-1977, 1949-1978, 19501979 ...1965-1994. For obvious reasons, the variations in $A_{p}$ within each interval were different and changed from a decrease in $A_{p}$ with time during the early intervals to an increase in $A_{p}$ during the later intervals. Figure 1 shows the different character of the $A_{p}$ variations within various inter-
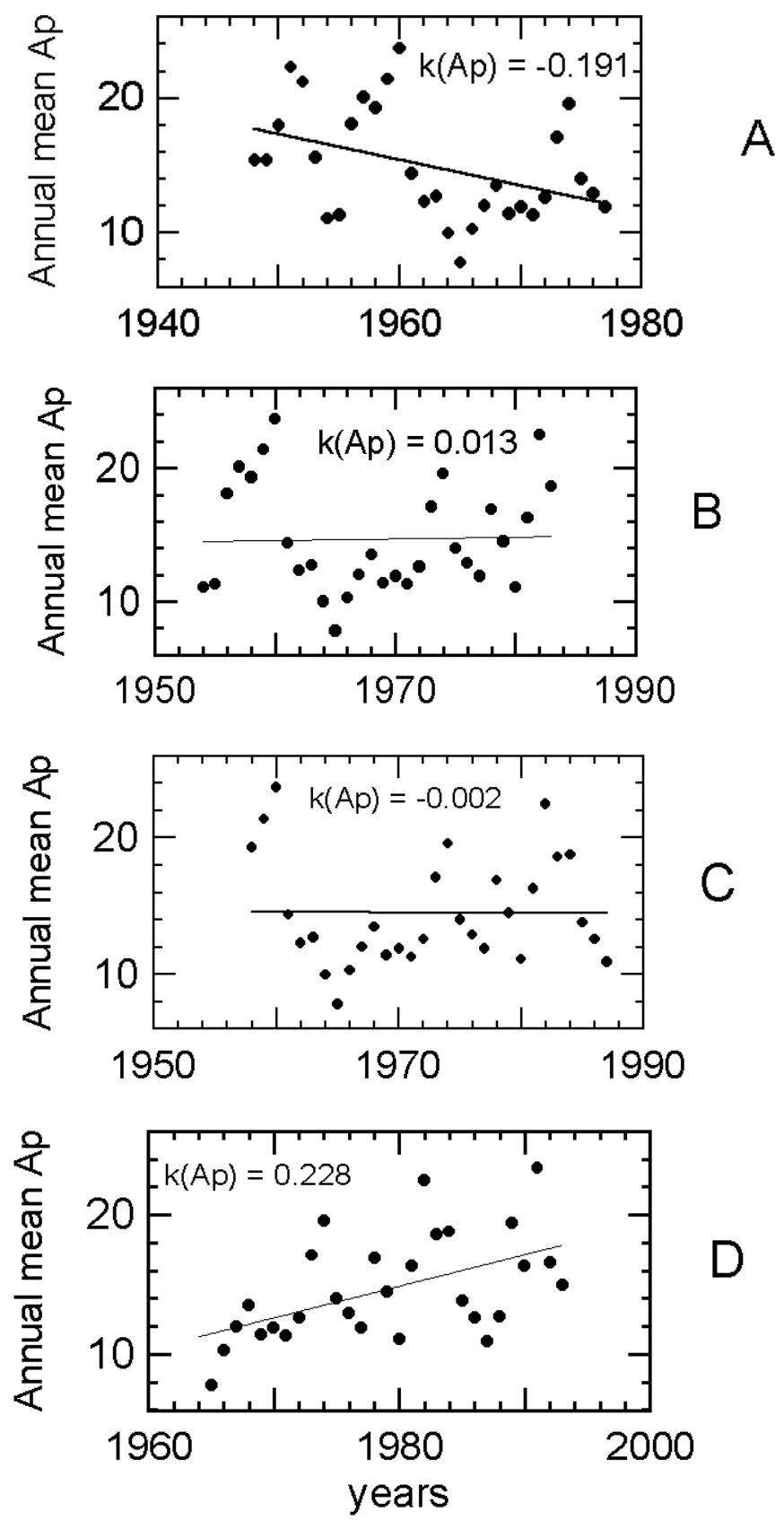

Fig. 1. Variation of the annual mean value of $A_{p}$ during: (a) the first 30-year interval 1948-1977, two intervals in the middle of the period considered (b) 1954-1977 and (c) 1958-1987, and (d) the last interval 1965-1994.

vals of the period considered. Panel (A) corresponds to the 1948-1987 interval, typical for the early intervals, when $A_{p}$ decreased with time. Panels (B) and (C) correspond to the 1954-1983 and 1958-1987 intervals, which are typical for 7 intervals when the systematic change in $A_{p}$ within intervals was small. Panel (D) corresponds to the 1965-1994 interval, typical for the later intervals when $A_{p}$ increased with time.

To describe the $A_{p}$ changes in each 30 -year interval, we 
Table 1. The annual mean values of the $A_{p}$ index for the years considered in this paper

\begin{tabular}{lcccccccccccccccc}
\hline Year & 1948 & 1949 & 1950 & 1951 & 1952 & 1953 & 1954 & 1955 & 1956 & 1957 & 1958 & 1959 & 1960 & 1961 & 1962 & 1963 \\
$A_{p}$ & 15.4 & 15.4 & 18.0 & 22.3 & 21.2 & 15.6 & 11.1 & 11.3 & 18.1 & 20.1 & 19.3 & 21.4 & 23.7 & 14.4 & 12.3 & 12.7 \\
\hline Year & 1964 & 1965 & 1966 & 1967 & 1968 & 1969 & 1970 & 1971 & 1972 & 1973 & 1974 & 1975 & 1976 & 1977 & 1978 & 1979 \\
$A_{p}$ & 10.0 & 7.8 & 10.3 & 12.0 & 13.5 & 11.4 & 11.9 & 11.3 & 12.6 & 17.1 & 19.6 & 14.0 & 12.9 & 11.9 & 16.9 & 14.5 \\
\hline Year & 1980 & 1981 & 1982 & 1983 & 1984 & 1985 & 1986 & 1987 & 1988 & 1989 & 1990 & 1991 & 1992 & 1993 & 1994 & \\
$A_{p}$ & 11.1 & 16.3 & 22.5 & 18.6 & 18.8 & 13.8 & 12.6 & 10.9 & 12.7 & 19.4 & 16.3 & 23.4 & 16.6 & 15.0 & 18.2 & \\
\hline
\end{tabular}

used the coefficient $k\left(A_{p}\right)$ of the $A_{p}$ linear regression within the interval considered: $A_{p}(X)=A_{p}\left(X_{1}\right)+k\left(A_{p}\right)\left(X-X_{1}\right)$, where $X_{1}$ is the first year of the interval in question and $X$ is the current year. Actually, $k\left(A_{p}\right)$ is merely the slope of the linear approximation of the $A_{p}$ value (lines in Fig. 1) plotted versus the years of the given interval.

It should be evident from the statements above that the values of $k\left(A_{p}\right)$ are negative for the early intervals, manifesting the tendency of $A_{p}$ to decrease, and positive for the later intervals when $A_{p}$ increases with time. Quantitatively, this fact is well illustrated by Table 2 . Below we will see how the trends in $f o \mathrm{~F} 2$ observed for each interval, $k$ (obs), change with changes in $k\left(A_{p}\right)$.

We consider here the relative trends $\delta f o \mathrm{~F} 2$ which are deviations of the observed $f o \mathrm{~F} 2$ from the model (a third-degree polynomial in terms of the smoothed solar activity index). The method has been proposed by Danilov and Mikhailov (1998) and then used in further publications on revealing trends and looking for their relation to geomagnetic activity (Danilov and Mikhailov, 1999, 2001; Mikhailov and Marin, 2000, 2001). For a more detailed description of the method and details of the $k$ (obs) variations observed (with geomagnetic latitude, local time etc.), we refer the readers to the publications indicated. We only note here that, as it has been shown in the above indicated papers, since the diurnal behaviour of $k(\mathrm{obs})$ is more pronounced than the seasonal one (the latter fact may be due to the use of smoothed values of $f o \mathrm{~F} 2$ to find $\delta f o \mathrm{~F} 2$ ), we consider below the annual mean values of $\delta f o \mathrm{~F} 2$ for various LT moments.

The only methodical difference of this paper from the papers mentioned above is that in this case, we did not use the sunspot number R12, but rather the E81 index based on the observations of the solar UV radiation, proposed by Tobiska et al. (2000). From general considerations one would expect this index to be more pertinent for describing ionospheric variations, though the monthly mean values of E81 $(r>0.95)$ correlate well with the monthly mean values of $R$; therefore, the change in the solar index could not influence significantly the results of this paper.

\section{Method I}

Now we consider in detail how the slope $k$ (obs) is determined as the trend $\delta f o \mathrm{~F} 2$ is formed. Evidently, if there is no systematic (longer than one cycle of solar activity) change in $A_{p}$, the $A_{p}$ influence on $f o \mathrm{~F} 2$, and nongeomagnetic trends, then the $f o \mathrm{~F} 2$ deviations from the model would have been of a random character and on the whole, the values of $\delta f o F 2$ would have been located along a horizontal line with some scatter caused by the measurement errors. In reality, as it is described in detail in the papers mentioned, the $\delta f o \mathrm{~F} 2$ are located along some line (since a linear approximation of $\delta f o \mathrm{~F} 2$ is considered) having a slope which is $k(\mathrm{obs})$.

If the changes in $\delta f o \mathrm{~F} 2$ were determined by the influence of geomagnetic activity only, then the slope $k$ (obs) would have been equal to either $a_{1} k\left(A_{p}\right)$ (if there is a direct relation between $A_{p}$ and $f o \mathrm{~F} 2$, i.e. $f o \mathrm{~F} 2$ grows with $A_{p}$ ) or $-a_{1} k\left(A_{p}\right)$ (if there is an inverse relation between $A_{p}$ and $f o \mathrm{~F} 2$ ). We will come to the $a_{1}$ coefficient below.

As an example, Table 2 shows the $k$ (obs) values for 10:00 LT for all 30-year intervals. Since the $A_{p}$ and $\delta f o F 2$ values have different absolute values, one obtain different by absolute value $k\left(A_{p}\right)$ and $k(\mathrm{obs})$. The problem of looking for the $a_{1}$ coefficient, which makes it possible to use jointly $k\left(A_{p}\right)$ and $k(\mathrm{obs})$, will be considered further. Just for the convenience of comparison of the time behaviour of $k(\mathrm{obs})$ and $k\left(A_{p}\right)$, we present in Table 2 the value of $k\left(A_{p}\right) / 100$ (that means that we arbitrarily took $a_{1}=0.01$ ) which, by its absolute value, has the same order as $k$ (obs).

Table 2 clearly demonstrates that the time behaviour (from one 30-year interval to another) of the $k(\mathrm{obs})$ and $k\left(A_{p}\right)$ values differs considerably. The $k$ (obs) is negative (with a relatively small change in the absolute value) for all the intervals considered, whereas $k\left(A_{p}\right)$ is negative for the first 11 intervals, with the absolute values changing by more than an order of magnitude from the 1948-1977 interval to the 1953-1982 and 1958-1987 intervals. For the intervals after 1959, $k\left(A_{p}\right)$ is positive, with the absolute value changing again by an order of magnitude from the 1959-1988 interval to the latest 4 intervals.

The above indicated difference in the behaviour of $k(\mathrm{obs})$ and $k\left(A_{p}\right)$ from one 30-year interval to another is observed to a greater or lesser degree than for the other LT moments. The existence of such a strong difference does not allow us 
Table 2. The $k$ values (Sverdlovsk, 10:00 LT)

\begin{tabular}{|c|c|c|c|c|c|c|}
\hline \multirow{2}{*}{$\begin{array}{l}\text { Time } \\
\text { Intervals }\end{array}$} & \multirow{2}{*}{$k\left(A_{p}\right)$} & \multirow{2}{*}{$k\left(A_{p}\right) / 100$} & \multirow[t]{2}{*}{$k(\mathrm{obs})$} & \multicolumn{3}{|c|}{$k(t r)$ for various $a_{1}$ values } \\
\hline & & & & 0.0010 & 0.0017 & 0.0020 \\
\hline 1948-77 & -0.191 & -0.00191 & -0.00128 & -0.00147 & -0.00160 & -0.00166 \\
\hline $1949-78$ & -0.175 & -0.00175 & -0.00120 & -0.00138 & -0.00150 & -0.00156 \\
\hline 1950-79 & -0.176 & -0.00176 & -0.00086 & -0.00104 & -0.00116 & -0.00122 \\
\hline $1951-80$ & -0.180 & -0.00180 & -0.00001 & -0.00019 & -0.00032 & -0.00037 \\
\hline $1952-81$ & -0.118 & -0.00118 & -0.00054 & -0.00066 & -0.00074 & -0.00078 \\
\hline 1953-82 & -0.020 & -0.00020 & -0.00075 & -0.00067 & -0.00078 & -0.00079 \\
\hline $1954-83$ & 0.013 & 0.00013 & -0.00083 & -0.00082 & -0.00081 & -0.00081 \\
\hline 1955-84 & 0.014 & 0.00014 & -0.00134 & -0.00133 & -0.00132 & -0.00132 \\
\hline $1956-85$ & -0.018 & -0.00018 & -0.00139 & -0.00141 & -0.00142 & -0.00143 \\
\hline $1957-86$ & -0.013 & -0.00013 & -0.00145 & -0.00146 & -0.00147 & -0.00147 \\
\hline $1958-87$ & -0.020 & -0.00020 & -0.00130 & -0.00132 & -0.00133 & -0.00134 \\
\hline 1959-88 & 0.019 & 0.00019 & -0.00120 & -0.00118 & -0.00117 & -0.00116 \\
\hline 1960-89 & 0.101 & 0.00101 & -0.00151 & -0.00141 & -0.00134 & -0.00131 \\
\hline 1961-90 & 0.179 & 0.00179 & -0.00173 & -0.00155 & -0.00143 & -0.00137 \\
\hline 1962-91 & 0.243 & 0.00243 & -0.00159 & -0.00135 & -0.00118 & -0.00111 \\
\hline 1963-92 & 0.244 & 0.00244 & -0.00143 & -0.00119 & -0.00102 & -0.00095 \\
\hline 1964-93 & 0.235 & 0.00235 & -0.00147 & -0.00123 & -0.00107 & -0.00099 \\
\hline 1965-94 & 0.228 & 0.00228 & -0.00139 & -0.00116 & -0.00100 & -0.00093 \\
\hline \multicolumn{2}{|c|}{$r\left[k(t r), k\left(A_{p}\right)\right]$} & & & -0.343 & -0.038 & 0.107 \\
\hline \multirow{2}{*}{\multicolumn{2}{|c|}{$k\left(t r\right.$, ave $\left._{1}\right)$}} & & & -0.00116 & -0.00115 & -0.00114 \\
\hline & & & & 0.00035 & 0.00034 & 0.00033 \\
\hline
\end{tabular}

to consider the observed variations in $\delta f o \mathrm{~F} 2$ with time (i.e. $k(\mathrm{obs}))$ as a result of the variations in $A_{p}$ only and it encourages us to assume that there is some other component that contributes to $k$ (obs) and is independent of $A_{p}$. This component is the very trend independent of $A_{p}$, we are searching for in this paper.

Now we consider the relation between $k\left(A_{p}\right), k(\mathrm{obs})$ and the nongeomagnetic trend $k(t r)$ that we are looking for. If, as we have assumed in the beginning, there are no other factors influencing the long-term variations in $f o \mathrm{~F} 2$ (the solar activity impact we have taken into account determining $\delta f o \mathrm{~F} 2$ ), the change in $\delta f o \mathrm{~F} 2, k(\mathrm{obs})$, observed within each 30 -year interval should be determined only by $k\left(A_{p}\right)$ and $k(t r)$. The formulae relating these three parameters would be different in the cases when an increase in $A_{p}$ leads to a decrease or increase in $f o \mathrm{~F} 2$, i.e. when the correlation coefficient $r$ between $A_{p}$ and $\delta f o \mathrm{~F} 2$ is negative or positive, respectively. For the majority of the LT moments, $r\left(\delta f o \mathrm{~F} 2, A_{p}\right)<0$. This fact is a principal argument in favor of the negative phases of ionospheric storms as a primary mechanism of the realization of the $A_{p}$ change influence on the $f o \mathrm{~F} 2$ trends (see above). $r\left(A_{p}, \delta f o \mathrm{~F} 2\right)$ has a small positive value only during a short LT interval around 16:00-18:00 LT.

Figure 2 illustrates the situation when $r\left(A_{p}, \delta f o \mathrm{~F} 2\right)<0$. For both the $A_{p}$ points and $\delta f o \mathrm{~F} 2$ points, a linear approximation is used. It is obvious that, if there was no other influence on $k$ (obs) except that of magnetic activity, then $a_{1} k\left(A_{p}\right)$ and $k$ (obs) should coincide. We remind ourselves that $a_{1}$ is a scaling coefficient (the same for all the 30-year intervals for the given LT) which makes possible a comparison of both slopes in codimensional units. If there exists a trend independent of $A_{p}$ (in Fig. 2, it is shown as negative), then the line $k$ (obs) would go below the $-a_{1} k\left(A_{p}\right)$ line by the $k(t r)$ value.

Thus, the formula relating all three values is simple:

$k(\mathrm{obs})=-a_{1} k\left(A_{p}\right)+k(t r)$,

or:

$k(t r)=k(\mathrm{obs})+a_{1} k\left(A_{p}\right)$.

Evidently, formulae (1) and (2) stay correct under any signs of the initial values $k\left(A_{p}\right)$ and $k(\mathrm{obs})$. It is only required that the condition $r\left(A_{p}, \delta f o \mathrm{~F} 2\right)<0$ be fulfilled.

When $r\left(A_{p}, \ddot{e} f o \mathrm{~F} 2\right)>0$ (i.e. under positive relation between $A_{p}$ and $f o \mathrm{~F} 2$ ), the term $a_{1} k\left(A_{p}\right)$ in Eq. (1) changes sign (for the case of simplicity, we take the $a_{1}$ coefficient to be positive always). In this case,

$k(\mathrm{obs})=a_{1} k\left(A_{p}\right)+k(t r)$

and

$k(t r)=k(\mathrm{obs})-a_{1} k\left(A_{p}\right)$.

Formulae (2) and (4) give us the first method to find $k(t r)$ from the observed values of $k(\mathrm{obs})$ for the given 30-year interval and the known values of $k\left(A_{p}\right)$, if we know the value of $a_{1}$. 


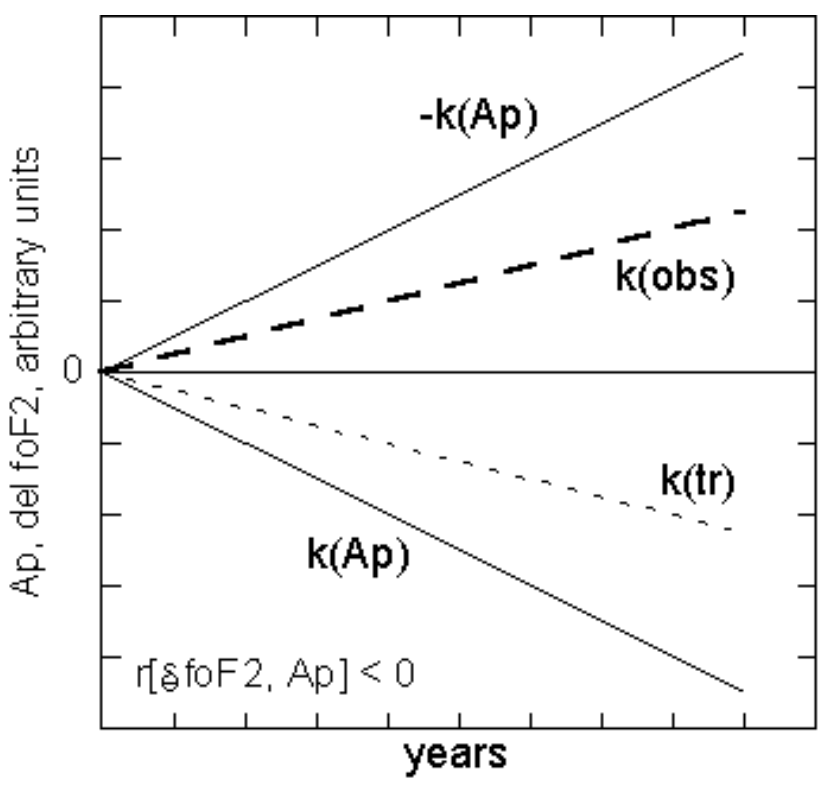

Fig. 2. The relation between the slope of various lines at the negative relation between $\delta f o \mathrm{~F} 2$ and $A_{p}$.

The value of $a_{1}$ is determined by two factors: scaling and physical. The scaling factor is due to different scales of the initial values of $A_{p}$ and $\delta f o \mathrm{~F} 2$. The physical factor depends on how a change in $A_{p}$, say, by a factor of two, changes (depletes if $r\left(A_{p}, \delta f o \mathrm{~F} 2\right)<0$ and enhances if $\left.r\left(A_{p}, \delta f o \mathrm{~F} 2\right)>0\right) \delta f o \mathrm{~F} 2$. Evidently, the resulting change should not be obligatory by a factor of two, but may be by a factor of 1.5 , by $30 \%$, by $10 \%$ etc. For our consideration here it is important only that such a relation (negative or positive) between $A_{p}$ and $\delta f o \mathrm{~F} 2$ does exist.

Therefore, one cannot find the $a_{1}$ coefficient independently based only on physical considerations. We have found the only way to determine $a_{1}$ which is the following. For every LT moment we have split (as it has been described above) the entire observational period (1948-1994) into eighteen 30 -year intervals. For each of these intervals we calculate step-by-step the $k(t r)$ values using formulae (2) or (4) and various values of $a_{1}$ (however, the same for all the 30-year intervals) and choose the value of $a_{1}$ that provides the lowest correlation between $k(t r)$ for each 30-year interval and the corresponding $k\left(A_{p}\right)$. The value of $k(t r)$ obtained for this value of $a_{1}$ is the very trend we are looking for. We remind ourselves that we are looking for a nongeomagnetic trend, i.e. the trend which is not related to the changes in $A_{p}$. One can see in Table 2 that the value of $k\left(A_{p}\right)$ changes dramatically from the early intervals to the later and even changes sign. If, nevertheless, the correlation coefficient $r\left[k(t r), k\left(A_{p}\right)\right]$ taken over all 18 lines of Table 2 is small (close to zero), then we may believe that we have found the value of $k(t r)$ that does not depend on magnetic activity.

The right-hand part of Table 2 shows an example of various $k(t r)$ obtained from the initial values of $k(o b s)$ and
$k\left(A_{p}\right)$ (shown in the left-hand part of Table 2) if different values of $a_{1}$ (three columns) are used. At the bottom of each column, the correlation coefficient $r\left[k(t r), k\left(A_{p}\right)\right]$, the value of $k\left(t r, a_{1} e_{1}\right)$ averaged over all 18 lines of the column and the corresponding standard deviation $\sigma$ of the $k\left(t r\right.$, ave $\left._{1}\right)$ value obtained are shown. It is worth noting that, since in this paper we use various levels of averaging, we use the designations $k\left(t r, \mathrm{ave}_{1}\right), k\left(t r, \mathrm{ave}_{2}\right)$ and so on. In the case under consideration, $k\left(t r\right.$, ave $\left._{1}\right)$ means averaging over all eighteen 30-year intervals for fixed LT.

Table 2 shows that the $k\left(t r\right.$, ave $\left.e_{1}\right)$ values obtained weakly depend on the $a_{1}$ value and so possible uncertainty in the $a_{1}$ determination would not influence significantly the final conclusion on the nongeomagnetic trend value. This statement is true for other LT moments as well. The close to zero value of the correlation coefficient $r\left[k(t r), k\left(A_{p}\right)\right]$ is reached in Table 2 at $a_{1}=0.0017$. The corresponding values of $k\left(t r\right.$, ave $\left._{1}\right)$ and $\sigma$ are -0.00115 and 0.00038 , respectively. It is important that with this $a_{1}$ value we also obtain a close to zero correlation coefficient of $k(t r)$ with time (with the year of the middle of each interval C). For example, for the column $a_{1}=0.0017$, the $r[k(t r), C]$ correlation coefficient is -0.05 . For other LT moments this coefficient is also small, so the trend found from the condition $r\left[k(t r), k\left(A_{p}\right)\right]=0$ has no significant changes with time within the period under consideration (1948-1994).

Thus, the method considered (we will call it Method I) makes it possible to obtain for the 30-year intervals considered the values of $k(t r)$ that correlate neither with the $A_{p}$ trend for the given interval, nor with time (the year of the interval middle). Obviously, it is the very nongeomagnetic trend that we are looking for in this paper.

\section{Method II}

To check our system on the whole and Method I in particular, we now consider a different method of $k(t r)$ determination for the same eighteen 30-year intervals. The method is as follows. We are able to add to the observed values of $\delta f o \mathrm{~F} 2$ within the given 30-year interval some trend $\Delta k$ such that the correlation coefficient between $\delta f o \mathrm{~F} 2$ and $A_{p}$ within the interval was maximum by the magnitude, i.e. that we turn the line $k$ (obs) by some angle $\Delta k$, achieving the maximum correlation coefficient between the values of $(\delta f o \mathrm{~F} 2+\Delta k)$ and $A_{p}$. We have already noted above that the correlation coefficient would be maximum when the resulting line coincides with $a_{2} k\left(A_{p}\right)$, if there is a positive relation between $f o \mathrm{~F} 2$ and $A_{p}$, and with $-a_{2} k\left(A_{p}\right)$, if the relation is negative. Figure 3 illustrates the latter case. Therefore:

$k(\mathrm{obs})+\Delta k=-a_{2} k\left(A_{p}\right)$.

If the relation of $f o \mathrm{~F} 2$ to $A_{p}$ is positive, a similar formula would be:

$k(\mathrm{obs})+\Delta k=a_{2} k\left(A_{p}\right)$. 


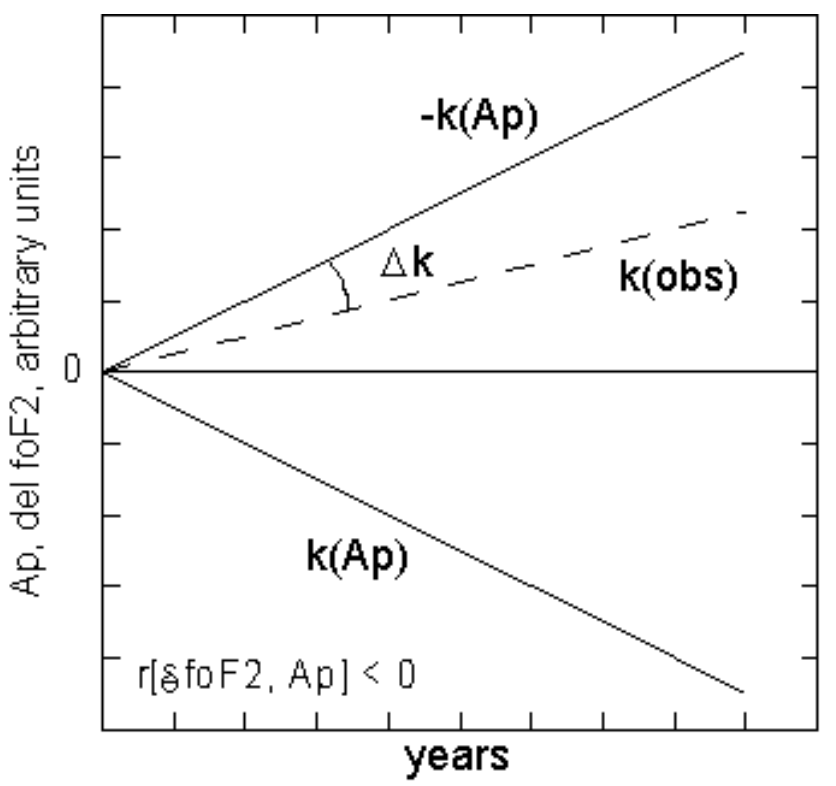

Fig. 3. Determination of the $\Delta k$ value at the negative relation between $\delta f o \mathrm{~F} 2$ and $A_{p}$.

It is worth noting that, in spite of the visual similarity of Methods I and II and formulae (3), (4), (5), and (6), the values of $a_{1}$ and $a_{2}$ are significantly different. The $a_{2}$ value is merely a coefficient equalizing the scales in which $k$ for $\delta f o \mathrm{~F} 2$ and $A_{p}$ is counted, whereas the $a_{1}$ value includes this coefficient and (it has been noted above) the coefficient indicating how the value of $\delta f o \mathrm{~F} 2$ changes for the given change in $A_{p}$. The latter coefficient, due to obvious reasons, is less than unity, so $a_{1}$ should be less than $a_{2}$. Below we will show examples supporting this statement and show how the values of $a_{1}$ and $a_{2}$ obtained by the two methods agree between themselves.

Figure 4 shows examples of variations of $r[(\delta f o \mathrm{~F} 2+$ $\left.\Delta k), A_{p}\right]$ versus $\Delta k$ in the vicinity of the maximum of $r$. One can see that $r$ changes smoothly, the maximum is well pronounced, and thus, the value of $\Delta k$ in each case may be determined fairly reliably.

Combining Eq. (3) with Eq. (5) and Eq. (4) with Eq. (6), we obtain:

$-k(t r)=\Delta k+\left(a_{2}-a_{1}\right) k\left(A_{p}\right)$

for $r\left(A_{p}, \delta f o \mathrm{~F} 2\right)<0$ and

$-k(t r)=\Delta k-\left(a_{2}-a_{1}\right) k\left(A_{p}\right)$

for $r\left(A_{p}, \delta f o \mathrm{~F} 2\right)>0$. Formulae (7) and (8) are the basis of Method II.

In the same way as in Method I, there is only one possibility in Method II to find the value of $\left(a_{2}-a_{1}\right)$. It is to find the value of $a_{2}-a_{1}$ (the same for all the 30-year intervals for the given LT) which provides the minimum correlation between the $k(t r)$ obtained and $A_{p}$ changes from one 30-year interval to another, i.e. $r\left[k(t r), k\left(A_{p}\right)\right]=0$.

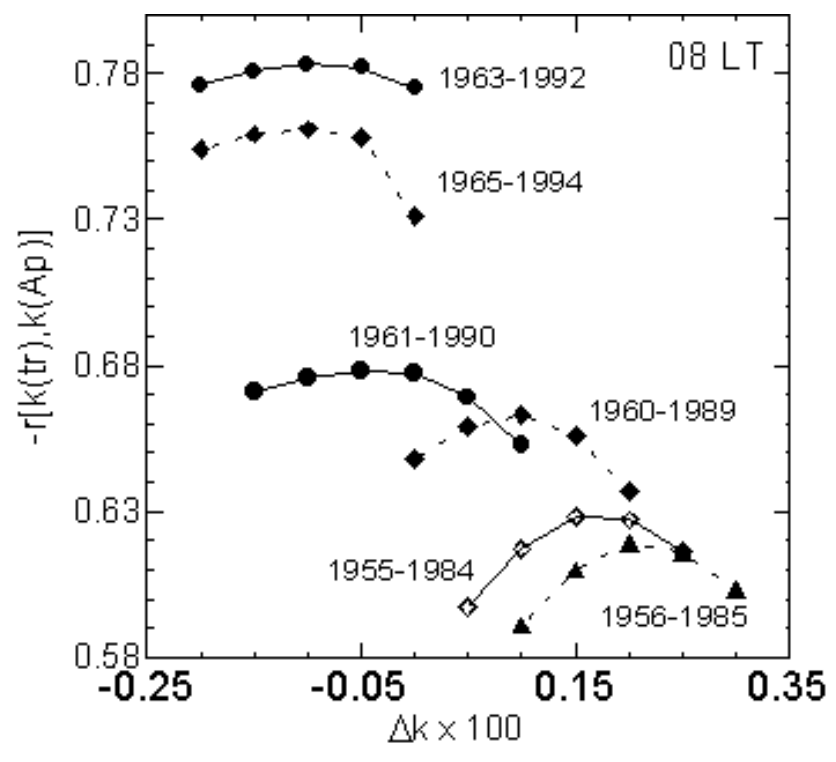

Fig. 4. Variations of the $r\left[k(t r), k\left(A_{p}\right)\right]$ value as a function of $\Delta k$ in the vicinity of the $r\left[k(t r), k\left(A_{p}\right)\right]$ maximum.

Table 3. The correlation coefficient $r\left[(k(o b s)+\Delta k), k\left(A_{p}\right)\right]$ for various LT moments

\begin{tabular}{lccccccc}
\hline LT & 00 & 02 & 04 & 06 & 08 & 10 & 22 \\
$r$ & -0.954 & -0.996 & -0.997 & -0.997 & -0.994 & -0.974 & -0.980 \\
\hline
\end{tabular}

Table 4. Values of $k(t r)$ and $\sigma$ for various LT moments

\begin{tabular}{cccccc}
\hline LT & \multicolumn{2}{c}{ Method I } & \multicolumn{2}{c}{ Method II } & Average \\
& $k\left(t r, \mathrm{ave}_{1}\right)$ & $\sigma$ & $k\left(t r, \mathrm{ave}_{1}\right)$ & $\sigma$ & $k\left(t r, \mathrm{ave}_{2}\right)$ \\
\hline 00 & -0.00132 & 0.00047 & -0.00173 & 0.00117 & -0.00152 \\
02 & -0.00099 & 0.00045 & -0.00115 & 0.00065 & -0.00107 \\
04 & -0.00123 & 0.00042 & -0.00141 & 0.00059 & -0.00132 \\
06 & -0.00130 & 0.00048 & -0.00116 & 0.00048 & -0.00123 \\
08 & -0.00168 & 0.00042 & -0.00177 & 0.00049 & -0.00173 \\
10 & -0.00115 & 0.00034 & -0.00152 & 0.00062 & -0.00133 \\
22 & -0.00121 & 0.00051 & -0.00155 & 0.00092 & -0.00138 \\
\hline
\end{tabular}

Calculating $k(t r)$ by Method II we have an additional method of control. In this method we add to the observed $\delta f o \mathrm{~F} 2$ values the corresponding values $\Delta k$, achieving the maximum correlation between the $(\delta f o \mathrm{~F} 2+\Delta k)$ values obtained and $A_{p}$. We expect that the slope $k(o b s)+\Delta k$ obtained coincides with $-a_{2} k\left(A_{p}\right)$, if the correlation between $\delta f o \mathrm{~F} 2$ and $A_{p}$ is negative, and with $a_{2} k\left(A_{p}\right)$, if it is positive. If this is true, the correlation coefficient between the values $k(o b s)+\Delta k$ and $a_{2} k\left(A_{p}\right)$ should be high. Actually, one 
Table 5. The values of the correlation coefficient $r\left(\delta f o \mathrm{~F} 2, A_{p}\right)$ for various LT moments $(r(1) 1948-1994 ; r(2) 1953-1988)$

$\begin{array}{lcccccccccccc}\text { LT } & 22 & 00 & 02 & 04 & 06 & 08 & 10 & 12 & 14 & 16 & 18 & 20 \\ r(1) & -0.22 & -0.33 & -0.39 & -0.41 & -0.34 & -0.42 & -0.35 & -0.13 & -0.08 & 0.10 & 0.05 & -0.03 \\ r(2) & -0.19 & -0.35 & -0.37 & -0.42 & -0.53 & -0.55 & -0.40 & -0.08 & -0.04 & 0.13 & 0.05 & -0.03\end{array}$

Table 6. The correlation coefficient $r\left(\delta f o \mathrm{~F} 2, A_{p}\right)$ for four LT moments for seven 30-year intervals with low values of the $k\left(A_{p}\right)$ magnitude

\begin{tabular}{lccccccc}
\hline int & $1953-82$ & $1954-83$ & $1955-84$ & $1956-85$ & $1957-86$ & $1958-87$ & $1959-88$ \\
\hline 02LT & -0.340 & -0.345 & -0.344 & -0.327 & -0.349 & -0.419 & -0.449 \\
08LT & -0.493 & -0.518 & -0.570 & -0.531 & -0.526 & -0.581 & -0.629 \\
20LT & -0.009 & 0.009 & -0.025 & 0.008 & 0.001 & -0.086 & -0.113 \\
14LT & -0.006 & 0.022 & -0.052 & -0.017 & -0.019 & -0.079 & -0.112 \\
\hline
\end{tabular}

can see in Table 3 that the value of $r\left[(k(o b s)+\Delta k), k\left(A_{p}\right)\right]$ for all LT moments for which Method II was used exceeds 0.95 and in many cases is almost equal to unity. This confirms the fact, that in reaching the maximum absolute value of the $r\left[(\delta f o \mathrm{~F} 2+\Delta k), A_{p}\right]$ correlation coefficient, we actually "turn" the observed line $k$ (obs) to the slope almost coinciding with $a_{2} k\left(A_{p}\right)$.

\section{Comparison of two methods}

The values of $k\left(t r\right.$, ave $\left._{1}\right)$ obtained for various LT moments by the two methods are shown in Table 4. The standard deviations $\sigma$ are also shown. The last column of Table 4 shows the values of $k\left(t r, \mathrm{ave}_{2}\right)$ averaged for the given LT over two methods. Table 4 shows that all values of $k(t r)$ obtained by the two methods are negative and their magnitude exceeds 0.001 . The $\sigma$ value do not exceed one-half of $k(t r)$ and in the majority of cases, it is about one-third of this value. The averaged values $k\left(t r, \mathrm{ave}_{2}\right)$ obtained for various LT moments of the period considered do not differ significantly from one another and lie within the interval from -0.00116 to -0.00173 , indicating no pronounced dependence on LT.

One can see from Table 4 that both methods in question have not been applied to all moments of the day, but only to the interval 22:00-10:00 LT. It is due to the following. Method I and Method II are based on withdrawing the effect of long-term variations related to geomagnetic activity. To perform such withdrawing reliably, one needs the relation of the initial values of $\delta f o \mathrm{~F} 2$ and $A_{p}$ to be well pronounced, i.e. the correlation coefficient $r\left(\delta f o \mathrm{~F} 2, A_{p}\right)$ should be stable by its sign and high enough by the magnitude. Danilov and Mikhailov (1999, 2001) showed that the value of $r\left(\delta f o \mathrm{~F} 2, A_{p}\right)$ depends on local time and is maximum in the nighttime and morning hours and minimum in the daytime and afternoon hours. This behaviour of $r\left(\delta f o \mathrm{~F} 2, A_{p}\right)$ is caused evidently by the features of the manifestation of the ionospheric storm negative phases at middle latitudes (for more details, see Danilov and Mikhailov, 2001; Mikhailov and Marin, 2000).

Table 5 shows the values of $r\left(\delta f o \mathrm{~F} 2, A_{p}\right)$ taken for the entire period considered (1948-1994) and for the period 19531988 when there is almost no systematic behaviour of $A_{p}$ over the years (see above) and the value of $r\left(\delta f o \mathrm{~F} 2, A_{p}\right)$ manifests pure correlation between the deviations of $f o \mathrm{~F} 2$ and $A_{p}$ from their mean values. Although Table 5 shows that there are no principal differences in these two coefficients, the $r$ values for 1953-1988 seem to be more representative for this study.

Comparing Tables 4 and 5, one can see that the methods of seeking $k(t r)$ discussed in this paper were successfully applied to the interval 22:00-10:00 LT, during which the value $r\left(\delta f o \mathrm{~F} 2, A_{p}\right)$ at least does not decrease below 0.2 by magnitude and mainly exceeds $0.3-0.5$. In the interval 12:00 20:00 LT, the values of $r\left(\delta f o \mathrm{~F} 2, A_{p}\right)$ are mainly less than 0.1 by magnitude, change sign from one LT moment to another, and are insignificant. The methods developed in this paper cannot be applied to this LT interval.

We will not go into details of the above fact and merely mention that at such low correlation coefficients, it is difficult to separate the components of $\delta f o \mathrm{~F} 2$ caused by the geomagnetic activity influence and nongeomagnetic trend. In this case, the values of $r\left(\delta f o \mathrm{~F} 2, A_{p}\right)$ for particular 30-year intervals we use in both methods are not only small by magnitude, but change their signs, thus making it impossible to determine the $a_{1}$ and $a_{2}$ values.

To illustrate the difference in behaviour of $r\left(\delta f o \mathrm{~F} 2, A_{p}\right)$ we show in Table 6 variations of this coefficient for two moments from the 22:00-10:00 LT period and two moments from the 12:00-20;00 LT period. Thus, both methods proposed have limitations and can be applied only in the LT period when there is a stable correlation between $\delta f o \mathrm{~F} 2$ and $A_{p}$, and $r\left(\delta f o \mathrm{~F} 2, A_{p}\right)$ does not change sign from one 30year interval to another. 
Table 7. Coefficients $a_{1}$ and $a_{2}$ calculated for various LT moments

\begin{tabular}{ccccc}
\hline LT & $a_{2}$ & $a_{1}$ & $\left(a_{2}-a_{1}\right)$ & $\left(a_{2}-a_{1}\right)_{F}$ \\
\hline 00 & 0.0302 & 0.0003 & 0.0299 & 0.0251 \\
02 & 0.0266 & $<0.00001$ & 0.0266 & 0.0266 \\
04 & 0.0218 & 0.0012 & 0.0206 & 0.0222 \\
06 & 0.0169 & 0.0005 & 0.0164 & 0.0165 \\
08 & 0.0157 & 0.0040 & 0.0117 & 0.0115 \\
10 & 0.0187 & 0.0017 & 0.0170 & 0.0160 \\
22 & 0.0323 & 0.0002 & 0.0321 & 0.0320 \\
\hline
\end{tabular}

Let us now come back to the $a_{1}$ and $a_{2}$ coefficients obtained by various methods. We determine $a_{1}$ in Method I. In Method II, we obtain the residual $\left(a_{2}-a_{1}\right)$ (see above formulae (7) and (8)). Moreover, taking the ratio $[k(o b s)+$ $\Delta k] / k\left(A_{p}\right)$ we are able (as it has been mentioned above, see also Table 3 ) to find $a_{2}$. If now we compare independently determined values $a_{1}, a_{2}$ and $\left(a_{2}-a_{1}\right)$, we would be able to estimate the internal agreement of the entire scheme considered in this paper.

The values of these coefficients are shown in Table 7 . Here the $a_{1}$ values are obtained in Method I by formula (2); $a_{2}$ values are determined from the $[k(o b s)+\Delta k] / k\left(A_{p}\right)$ ratio, and the value of $\left(a_{2}-a_{1}\right)$ in the fourth column is merely the residual of the two previous columns. The value of $\left(a_{2}-a_{1}\right)$ is determined in Method II by formula (7). By comparing the two right-hand columns in Table 7 , one can see that the values of the coefficients $a_{1}, a_{2}$, and $\left(a_{2}-a_{1}\right)$, determined completely independently, agree fairly well. It should be noted that the $a_{2}$ coefficient, which (as we have noted above) is determined only by the ratio of scales of $\delta f o \mathrm{~F} 2$ and $A_{p}$, changes with LT relatively weakly (less than by a factor of 2). At the same time, the $a_{1}$ coefficient is (as we have predicted above) much smaller than $a_{2}$ and changes much stronger; the changes evidently manifesting a different efficiency of the impact of $A_{p}$ changes on variations of $\delta f o \mathrm{~F} 2$ at different LT moments. It is worth emphasizing that the highest values of $a_{1}$ are obtained for 06:00-10:00 LT when one should expect the strongest manifestation of negative phases of ionospheric storms at the Sverdlovsk latitude.

\section{Discussion}

Thus, by using the two methods proposed above, we determined the values of the systematic trend of $f o F 2$ for 1948 1994. The principal feature of this trend is that it does not depend on the character of time changes in geomagnetic activity $k\left(A_{p}\right)$. For example, one can see from Table 2 that for three intervals that differ by the $A_{p}$ behaviour: $A_{p}$ decreases $\left(1959-1979, k\left(A_{p}\right)=-0.176\right), A_{p}$ almost does not change $\left(1959-1988, k\left(A_{p}\right)=0.019\right)$, and $A_{p}$ increases $\left(1962-1991, k\left(A_{p}\right)=0.243\right)$, one obtains approximately the same trend $(k(t r) \sim 0.00117)$. That is what makes it possi- ble to consider the trend derived as the nongeomagnetic trend to search for, which this paper is aimed to.

Reasonable agreement between the $k\left(t r, a_{2} e_{2}\right)$ obtained for each LT by both methods proposed may be considered as a confirmation of the correctness and internal agreement of the entire scheme considered and the accepted description of the relation between the observed values $k$ (obs), the character of the $A_{p}$ time variations $k\left(A_{p}\right)$, and the nongeomagnetic trend $k(t r)$ that we were looking for. The agreement between the coefficients $a_{1}, a_{2}$, and $a_{2}-a_{1}$ determined independently also presents such a confirmation.

All the above said leads to a conclusion that the nongeomagnetic trend derived for the 22:00-10:00 LT period is (if we average the values of $k\left(t r, \mathrm{ave}_{2}\right)$ over LT) $k\left(t r, \mathrm{ave}_{3}\right)=$ -0.00146 . Since the method used (for details, see Danilov and Mikhailov, 1998, 1999) gives a relative trend (i.e. the relative change in the parameter analyzed per year), in order to obtain the absolute trend, one should multiply the relative trend value by the annual mean value of the parameter. In our case, it is $f o \mathrm{~F} 2$ and we may accept for an approximate evaluation $f o \mathrm{~F} 2$ (annual mean) $=10 \mathrm{MHz}$. In this case, the value $k\left(t r\right.$, ave $\left._{3}\right)=-0.0015$ per year corresponds to the absolute trend of about $-0.015 \mathrm{MHz}$ per year.

The question as to whether there is a diurnal variation of the nongeomagnetic trend is not yet finally solved. Within the LT interval, when the methods proposed are applicable, no systematic dependence of $k\left(t r, \mathrm{ave}_{2}\right)$ on LT is seen. This made it possible to average the $k\left(t r, a_{2}\right)$ values to obtain $k\left(t r, \mathrm{ave}_{3}\right)=-0.0015$, which may be considered as a daily mean value of the nongeomagnetic trend at the Sverdlovsk station. The presence of a considerable LT interval in which the proposed methods cannot be applied leaves the question of the nongeomagnetic trend diurnal variations open. However, with the data from the Irkutsk station briefly considered below, one may think that (contrary to geomagnetic trends) there is no significant diurnal variation of the nongeomagnetic trend. This fact may be important for answering the question: long-term trends of which atmospheric parameters (pressure, temperature, composition, dynamics) cause the $f o \mathrm{~F} 2$ trends derived in this paper? It is obvious only that such a parameter should be the one that influences $f o \mathrm{~F} 2$ at night (and probably is weak in the daytime). In detail, the problem of the possible nature of the nongeomagnetic trend should be considered after application of the proposed methods to the data of many ionospheric stations of the global network, which is the subject of another study.

It is worth drawing attention to one fact which is not related directly to the search of nongeomagnetic trends, but rather helps to understand better how the correlation of $\delta f o \mathrm{~F} 2$ with geomagnetic activity is realized. One might have thought that the major input to this correlation is provided by a similarity of the slopes of the $\delta f o \mathrm{~F} 2$ and $A_{p}$ regression lines for the time interval considered. However, it is not the case.

Table 6 shows that for the 02:00 and 08:00 LT moments, even in the 30-year intervals within which the general tendency of $A_{p}$ variation is small ( $k\left(A_{p}\right)$ by magnitude is be- 


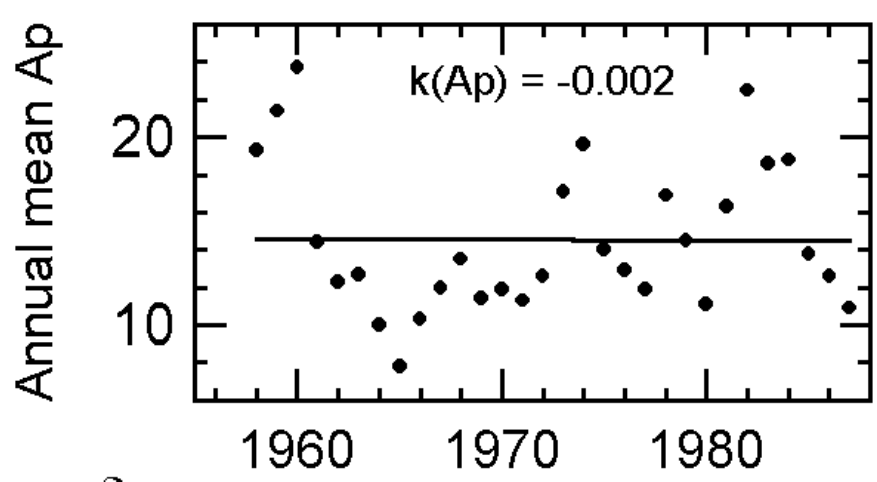

¿foF2

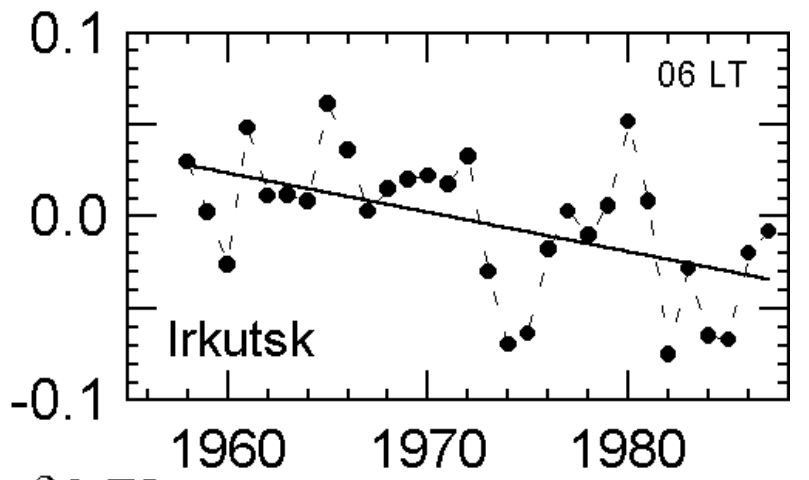

ffoF2
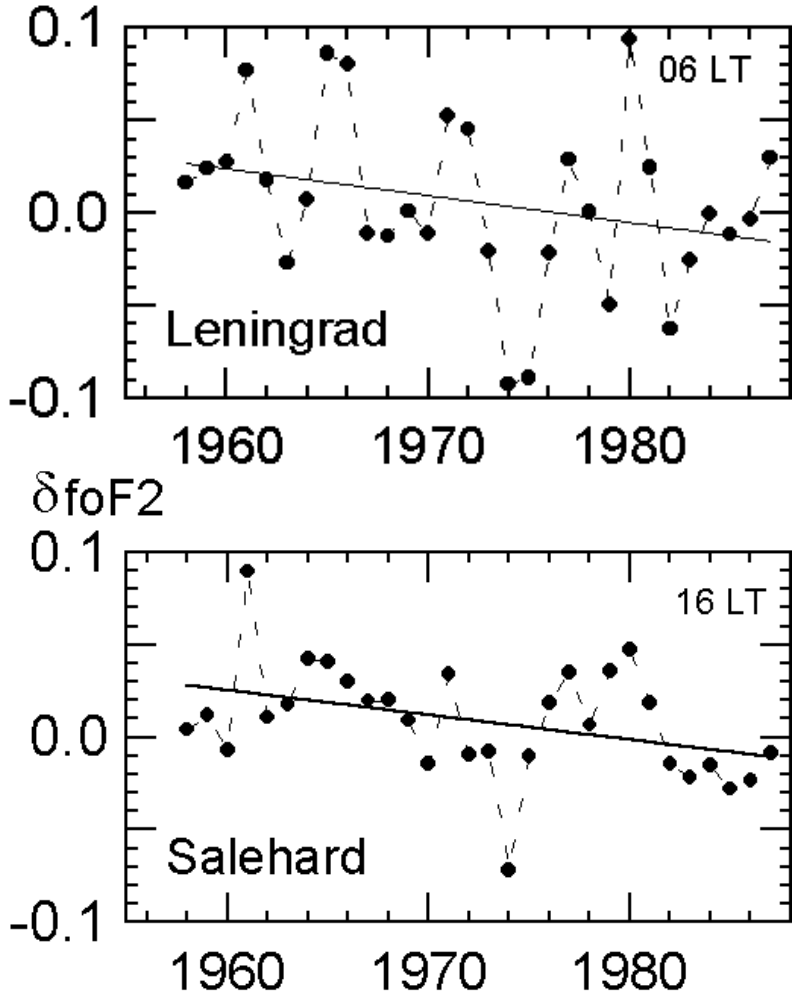

Fig. 5. Variations of $A_{p}$ (top panel) and $\delta f o \mathrm{~F} 2$ for three stations for the 1958-1987 period. (the values of $k$ (obs) for Irkutsk, Leningrad, and Salekhard are $-0.0011,-0.0014$, and -0.0013 per year, respectively)

low 0.02), a high (with the correlation coefficient in some moments reaching 0.7 ) correlation between the deviations of $\delta f o \mathrm{~F} 2$ from the regression lines and $A_{p}$ is observed.

Evidently (taking into account the absence of a significant $k\left(A_{p}\right)$ slope within the 7 intervals in question), the high correlation coefficient is provided by high correlation of the deviations of $\delta f o \mathrm{~F} 2$ from the regression model with $A_{p}$, or, to be exact, with the deviations of the $A_{p}$ value for each year from the regression line (of the type shown in Fig. 1) for each 30-year interval. Figure 1 shows that these deviations are large enough independent of whether there is or there is not a systematic $A_{p}$ change (increase or fall) within the given interval.

The fact described above shows that the correlation of the deviations of the annual mean values of $f o \mathrm{~F} 2$ and $A_{p}$ from corresponding regression lines plays a much larger role in the relation of $f o \mathrm{~F} 2$ trends to $A_{p}$ found in several papers (Danilov and Mikhailov, 1999, 2001; Mikhailov and Marin, 2000,2001 ) than long-term systematic changes in $A_{p}$ (for example, a systematic increase in $A_{p}$ from 1965 to 1961 mentioned above).

Figure 5 provides a visual example of the situation, when there is almost no trend in Ap for the 30-year interval chosen (1958-1987) but there exist pronounced and statistically significant trends in foF2 at various stations. Coming back to the Introduction, Fig. 5 provides a reason for looking for nongeomagnetic trends, because one for this particular interval can not obtain the high and statistically significant values of $k(\mathrm{obs})$, derived for many stations.

Though a detailed analysis of the nongeomagnetic trends at various stations is a matter of another publication, it is worth giving an example of the application of the approach proposed to another station. Since it has been shown above that Methods I and II agree well and that they confirm the correctness of the whole system relations between $k$ (obs), $k\left(A_{p}\right)$, and $k(t r)$, which is the basis for the entire consideration, we used only Method I as a more simple and visible approach. The Irkutsk ionospheric station was chosen $\left(\Phi=41^{\circ} \mathrm{N}, \varphi=52^{\circ} \mathrm{N} ; 1949-1991\right)$. The results of the analysis are shown in Table 8. The Irkutsk station is much more simple to analyze than the Sverdlovsk one. One can see from Table 8 that there are two periods of the day when there a stable positive and negative correlation between $\delta f o \mathrm{~F} 2$ and $A_{p}$ can be seen. As has been shown above, the presence of stable correlation is a necessary condition for using the methods developed in this paper.

Thus, the nongeomagnetic trend $k(t r)$ is reliably determined for 00:00-08:00 LT $\left(r\left(\delta f o \mathrm{~F} 2, A_{p}\right)<0\right.$, so Eq. 2 is used $)$ and 14:00-18:00 LT $\left(r\left(\delta f o \mathrm{~F} 2, A_{p}\right)>0\right.$, so Eq. 4 is used). The intermediate transition periods 10:00-12:00 LT and 20:00-22:00 LT are characterized by low values of $r\left(\delta f o \mathrm{~F} 2, A_{p}\right)$. What is more important during these $\mathrm{LT}$ is the fact that the $a_{1}$ coefficient changes sign from one 30-year interval to another, and so it is impossible to obtain $a_{1}$ and $k(t r)$. For the rest of the LT moments, both $k(t r)$ and $a_{1}$ are determined quite reliably. The values of $k(t r)$ obtained for the two LT periods (with positive and negative correlation 
Table 8. Determination of the $f o \mathrm{~F} 2$ trend for the Irkutsk station

\begin{tabular}{ccccccc}
\hline $\mathrm{LT}$ & $r\left(\delta f o \mathrm{~F} 2, A_{p}\right)$ & $r\left[k(o b s), k\left(A_{p}\right)\right]$ & $a_{1}$ & $r\left[k(t r), k\left(A_{p}\right)\right]$ & $k\left(t r, \mathrm{ave}_{1}\right)$ & $\sigma$ \\
\hline 00 & -0.13 & -0.19 & 0.0007 & 0.005 & -0.00105 & 0.00047 \\
02 & -0.29 & -0.55 & 0.002 & 0.012 & -0.00125 & 0.00044 \\
04 & -0.41 & -0.63 & 0.002 & 0.007 & -0.00140 & 0.00036 \\
06 & -0.75 & -0.53 & 0.0017 & 0.011 & -0.00181 & 0.00034 \\
08 & -0.29 & -0.50 & 0.002 & -0.003 & -0.00163 & 0.00045 \\
10 & -0.12 & 0.03 & 0.0001 & & & \\
12 & 0.03 & 0.35 & -0.0012 & & & \\
14 & 0.13 & 0.46 & -0.0015 & 0.008 & -0.00110 & 0.00038 \\
16 & 0.16 & 0.76 & -0.0026 & -0.010 & -0.00109 & 0.00034 \\
18 & 0.22 & 0.52 & -0.0035 & 0.011 & -0.00123 & 0.00038 \\
20 & 0.07 & 0.54 & & & & \\
22 & 0.02 & -0.19 & & & & $k\left(t r, \mathrm{ave}_{2}\right)=-0.00132$ \\
$r$ & $\left.r\left(\delta f o \mathrm{~F} 2, A_{p}\right), a_{1}\right]=-0.90617$ & & & & $\sigma$ & 0.00028
\end{tabular}

between $\delta f o \mathrm{~F} 2$ and $A_{p}$ ) all have negative signs and closed magnitudes. The averaged value of $k\left(t r, \mathrm{ave}_{2}\right)$ over these periods is -0.00132 , with $\sigma=0.00028$. One can see that the final values of the nongeomagnetic trends for two stations are close to each other, though there is a difference in geomagnetic latitudes.

Three features of the data in Table 8 should be noted. First, the periods of positive and negative correlation of $\delta f o \mathrm{~F} 2$ with $A_{p}$ qualitatively agree with the current ideas on the periods of appearance of negative and positive phases of an ionospheric storm. Thus, the results obtained in no way deny the hypothesis of magnetic activity trend influence on the resulting trend of $f o \mathrm{~F} 2$, but rather confirm it, providing at the same time a proof that there is a nongeomagneic trend, contrary to the geomagnetic trend showing no pronounced diurnal behaviour.

Second, in both cases $\left(r\left(\delta f o \mathrm{~F} 2, A_{p}\right)\right.$ positive and negative), a close to zero value of the correlation coefficient $r\left[k(t r), k\left(A_{p}\right)\right]$ is reached, demonstrating that the trend derived is really independent of $A_{p}$.

Third, there is a very high correlation coefficient between $a_{1}$ and $\delta f o \mathrm{~F} 2, A_{p}$, confirming the suggestion formulated in the beginning that $a_{1}$ includes not only the scaling factor as $a_{2}$, but the effectiveness of the magnetic activity impact on $f o \mathrm{~F} 2$ at each particular LT hour.

\section{Conclusions}

The attempt to analyze in detail the $f o \mathrm{~F} 2$ data at the Sverdlovsk station during 1948-1994 in order to clarify the question as to whether the trends found earlier (Danilov and Mikhailov, 1999; Mikhailov and Marin, 2000) are caused only by the long-term changes in geomagnetic activity, led to the following results.
Two methods to look for $f o \mathrm{~F} 2$ trends $k(t r)$ that demonstrate no visual relation to the long-term $A_{p}$-index behaviour, are developed. Both independent methods for seeking nongeomagnetic trends proposed in this paper give the results that agree well between themselves.

The values of the nongeomagnetic trend $k\left(t r, \mathrm{ave}_{2}\right) \mathrm{ob}-$ tained for the 22:00-10:00 LT period demonstrate no pronounced dependence on the time of the day and make it possible to obtain an average value $k\left(t r, \mathrm{ave}_{3}\right)=-0.0015$ per year (or $-0.015 \mathrm{MHz}$ per year if we take the annual mean value $f o \mathrm{~F} 2$ roughly equal to $10 \mathrm{MHz}$ ).

The methods developed here cannot be applied for the 12:00-20:00 LT interval when the correlation coefficient between $f o \mathrm{~F} 2$ and $A_{p}$ is small and it changes sign from one 30 -year interval to another. Therefore, there is still no final answer to the question as to whether there is or there is not a diurnal variation of the nongeomagnetic trend.

An example of application of Method I to the data of another station (Irkutsk) confirms all of the conclusions considered in detail for Sverdlovsk and gives the mean $f o \mathrm{~F} 2$ trend -0.00132 , which is close to the result for Sverdlovsk.

Acknowledgement. The author thanks Dr. K. Tobiska for the data on the E81 index and Prof. A. Mikhailov for the discussion of the paper.

Topical Editor M. Lester thanks M. Jarvis and another referee for their help in evaluating this paper.

\section{References}

Adushkin, V. V., Koslov, S. I., and Petrov, A. V.: Ecological problems and risks of the rocket and space technique impact on the environment (in Russian), Moscow, Ankil, 638, 2000.

Bencze, P., Sole, G., Alberca, L. F., and Poor, A.: Long-term changes of $h m \mathrm{~F} 2$ possible latitudinal and regional variations, Proc. of the 2nd COST 251 Workshop "Algorithms and Models 
for COST 251 Final Product”, 30-31 March, 1998, Side Turkey, Rutherford Appleton Lab.,UK, 107-113, 1998.

Bremer, J.: Some additional results of long-term trends in verticalincidence ionosonde data, Paper presented at the COST 251 Meeting, Prague, September 1996.

Bremer, J.: Trends in the ionospheric E and F regions over Europe, Ann. Geophysicae, 16, 986-996, 1998.

Bremer, J.: Trends in the thermosphere derived from global ionosonde observations, Adv. Space Res., accepted, 2001.

Danilov, A. D.: Long-term changes of the mesosphere and lower thermosphere temperature and composition, Adv. Space Res., 20, 2137-2147, 1997.

Danilov, A. D. and Mikhailov, A. V.: Long-term trends of the F2layer critical frequencies: A new approach, Proc. of the 2nd COST 251 Workshop "Algorithms and Models for COST 251 Final Product", 30-31 March, 1998, Side Turkey, Rutherford Appleton Lab., UK, 114-121, 1998.

Danilov, A. D. and Mikhailov, A. V.: Spatial and seasonal variations of the $f o$ F2 long-term trends, Ann. Geophysicae, 17, 1239$1243,1999$.

Danilov, A. D. and Mikhailov, A. V.: F2-layer parameter long-term trends at Argentine Islands and Port Stanley stations, Ann. Geophysicae, 19, 341-349, 2001

Givishvili, G. V. and Leshchenko, L. N.: Long-term trends of the properties of the midlatitude ionosphere and thermosphere, Dokl. RAN (in Russian), 333(1), 86-88, 1993.

Givishvili, G. V. and Leshchenko, L. N.: Possible proof of the presence of technogenic impact on the midlatitude ionosphere, Dokl. RAN (in Russian), 334(2), 213-214, 1994.

Jarvis, M. J., Jenkins, B., and Rogers, G. A.: Southern hemisphere observations of a long-term decrease in F-region altitude and thermospheric wind providing possible evidence for global thermospheric cooling, J. Geoph. Res., 103, 20 744-20 787, 1998.

Marin, D., Mikhailov, A. V., de la Morena, B. A., and Herraiz, M.:
Long-term $h m \mathrm{~F} 2$ trends in the Eurasian longitudinal sector on the ground-based ionosonde observations, (accepted to Ann. Geophys., 19, 761-772, 2001.

Mikhailov A. V. and Marin, D.: Geomagnetic control of the $f o \mathrm{~F} 2$ trends, Ann. Geophysicae, 18, 653-665, 2000.

Mikhailov, A. V. and Marin, D.: An interpretation of the $f o \mathrm{~F} 2$ and $h m \mathrm{~F} 2$ long-term trends in the framework of the geomagnetic control concept, Ann. Geophysicae, 19, 733-748, 2001.

Rishbeth, H.: A greenhouse effect in the ionosphere?, Planet. Space Sci., 38, 945-948, 1990.

Rishbeth, H. and Roble, R. G.: Cooling of the upper atmosphere by enhanced greenhouse gases - Modeling of thermospheric and ionospheric effects, Planet. Space. Sci., 40, 1011-1026, 1992.

Sergeenko, N. P. and Givishvili, G. V.: On the problem of manyyear properties of ionospheric disturbances, Geomagnetism and aeronomy (in Russian), 37(2), 108-112, 1997.

Sergeenko, N. P. and Kuleshova, V. P.: Climatic changes of the properties of disturbances in the ionosphere and upper atmosphere, Dokl. RAN (in Russian), 334, 534-537, 1994.

Tabiska, W. K., Woods, T. N., Eparvier, F. G., and Bouwer, S. D. Validation of the 10.7 proxy produced by SOLAR2000, Paper presented at the 33th COSPAR Scientific Assembly, Warsaw, (Abstracts, p. 89), 2000.

Ulich, T. and Turunen, E.: Evidence for long-term cooling of the upper atmosphere in ionospheric data, Geophys. Res. Lett., 24, 1103-1106, 1997.

Ulich, T., Karinen, A., and Turunen, E.: Effects of solar variability seen in long-term EISCAT radar observations of the lower ionosphere, Paper presented at the Second IAGA/CMA Workshop on Solar Activity Forcing of the Middle Atmosphere, Prague, August 1997.

Upadhyay, H. M. and Mahajan, K. K.: Atmospheric greenhouse effect and ionospheric trends, Geophys. Res. Lett., 25, 3375$3378,1998$. 